Article

\title{
Development of Terahertz Time-Domain Spectroscopy for Properties Analysis of Highly Diluted Antibodies
}

\author{
Nikita Penkov* (10) and Eugeny Fesenko \\ Institute of Cell Biophysics RAS, Federal Research Center Pushchino Scientific Center for Biological Research of \\ the Russian Academy of Sciences, Institutskaya str. 3, 142290 Pushchino, Russia; fessenko-ee@rambler.ru \\ * Correspondence: nvpenkov@rambler.ru
}

Received: 26 September 2020; Accepted: 30 October 2020; Published: 31 October 2020

check for

updates

Featured Application: Described in this work approach to THz-TDS method is applicable for analyzing structural properties of the solutions containing highly diluted antibodies and for the indirect investigation of altered water properties in aqueous solutions when direct measurements of a substance concentration are not available.

\begin{abstract}
This work represents the development of a three-band (Debye relaxation, intermolecular bending, and stretching vibrational bands) approach to the terahertz time-domain spectroscopy (THz-TDS) for the analysis of aqueous solutions of lactose saturated with highly diluted (HD) antibodies to interferon-gamma. Since THz-TDS is sensitive to a very slight changes in water properties, we aimed to assess its applicability for the analysis of HD antibodies. To evaluate water features in tested samples we also used Fourier transform infrared spectroscopy, considering the first overtone of intramolecular stretching vibrations of water molecules. We showed a broadening of the energy distribution of intermolecular bonds in the solution of HD substances in comparison to the control solution. This broadening corresponded to the presence of an active component even in HD samples. The results of both methods were similar; however, with the THz-TDS it was possible to analyze the changes in energy distribution of intermolecular (hydrogen) bonds in the solutions. Therefore, our approach to THz-TDS method is applicable for analyzing structural properties of the solutions containing HD antibodies and for the indirect investigation of altered aqueous properties in water solutions when direct measurements of a substance concentration are not available.
\end{abstract}

Keywords: terahertz spectroscopy; THz-TDS; high dilutions; solution structure; dynamics of water molecules

\section{Introduction}

Terahertz spectroscopy is a modern and rapidly developing method capable of analyzing the structure of matter. The terahertz region corresponds to intermolecular vibrations of molecules in terms of energy $(\mathrm{meV})$ and frequency $(\mathrm{THz})$ values which allows investigating the intermolecular structure and dynamics. The method has been successfully tested in various studies and has been proven to be applicable for addressing tasks in the fields of molecular biology [1-7], medicine [8,9], pharmacy [10-12], etc. However, in most of these works the studied objects were substances with low quantity of water, whereas this method has been rarely used to study aqueous solutions, for example in $[13,14]$. This is due to a strong absorption of the water itself which masks the absorption of dissolved substances.

Nevertheless, of particular interest are some areas of research exploiting terahertz spectroscopy which have made it possible to obtain previously unknown and fundamentally new data on aqueous 
solutions. In a number of studies aimed at analyzing aqueous solutions of various substances, in particular sugars and proteins, the molecules of these substances have been shown to have hydration shells 10 to $50 \AA$ thick [15-17] which corresponds to dozens of layers of water molecules. None of the previously known experimental approaches have made it possible to register such extended hydration shells. In another series of papers [18-22], terahertz spectroscopy was used for studying the structure and dynamics of molecules in various aqueous solutions which resulted in establishing quantitative relationships between the spectral parameters in the terahertz region and the fraction of free water molecules. Based on the obtained information, a novel method for the analysis of water structuredness has been proposed. These studies describe the features of spectra measurement which allow for the exclusion of a number of unwanted optical and spectral artifacts. As a result, it becomes possible to determine the dielectric function of aqueous solutions with high accuracy. In the article [23] a study of the effect of protein molecules in various conformations on the water environment has been described. The abovementioned results indicate a very high potential of the terahertz spectroscopy technique which generally makes it possible to study a wide range of objects with high water content.

Such objects include highly diluted solutions obtained by serial dilution technology with vigorous shaking at each stage. To date, more and more data on their effectiveness appear. In particular, it has been shown that high dilutions of antibodies can be used to treat various diseases [24-28]. High dilutions exert a modifying effect on the properties of the starting material [29-33], despite the colossal difference in the quantities of mixed substances (of the original substance and its high dilution). All this indicates that in the process of dilution the organization of hydration shells of molecules can change which determines the effects of high dilutions [34].

Unfortunately, the sensitivity limit of modern analytical methods does not allow for the direct detection of the active component in highly diluted solutions of substances. However, a THz technique can solve the problem as it allows to investigate the peculiarities of intermolecular bonds in solutions sensitive to changes in the composition of the solution. It is capable of providing information on the intermolecular structure and interactions in solutions in order to elucidate the physicochemical nature of the effects of the above-mentioned drugs.

One of the most up-to-date modifications of the method is the terahertz time-domain spectroscopy (THz-TDS) which offers wide opportunities for conducting reliable measurements of spectra in the 5-110 $\mathrm{cm}^{-1}$ region $(0.15-3.3 \mathrm{THz})$ [35-40]. THz-TDS allows us to simultaneously obtain both the absorption and refractive index spectra of the substance. These two functions make it possible to directly calculate the complex dielectric permittivity function of a substance which substantially increases the informational value of data as compared to conventional absorption spectra [36,41,42].

The decomposition of the dielectric function into components corresponding to various types of intermolecular dynamics of water is a very informative approach. However, there is still no unambiguous understanding of how many types of vibrational and relaxation dynamics of water molecules (how many spectral bands) must be taken into account when analyzing the spectra in the indicated terahertz region. Depending on the situation, it is required to determine the optimal number of model spectral bands, focusing on achieving highly informative data and, at the same time, its unambiguity.

The objective of this study was to adapt the THz-TDS technique so that to obtain reproducible and clearly interpretable information about the molecular structure and dynamics of solutions containing highly diluted antibodies.

\section{Materials and Methods}

\subsection{Tested Samples}

Three samples of lactose powder produced from lactose monohydrate (SuperTab ${ }^{\circledR}$ 30GR, DFE pharma, Goch, Germany) of the same batch were tested in the present study: 
(1) Lactose (lactose monohydrate) saturated with a solution of affinity purified rabbit polyclonal antibodies (Abs) to interferon gamma (IFN- $\gamma$ ) which had previously undergone a process of gradual reduction of their initial concentration. Briefly, Abs to IFN- $\gamma(2.5 \mathrm{mg} / \mathrm{mL})$ were diluted in water-ethanol solutions under specific conditions [43] with several steps of centesimal dilution. The first centesimal dilution was prepared by mixing 1 part of the original substance and 99 parts of the solvent. All subsequent dilutions contained 1 part of the previous dilution and 99 parts of the solvent. The final solution contained a mixture of 12,30 , and 50 centesimal dilutions of antibodies to IFN- $\gamma$ (highly diluted antibodies). The estimated theoretical Abs concentration in the final solution was no more than $2.5 \times 10^{-24} \mathrm{mg} / \mathrm{mL}$, or $2.5 \times 10^{-18} \mathrm{ppb}(\mathrm{w} / \mathrm{w})$. However, it should be noted that the physico-chemical studies showed that for the samples manufactured using the technology of high dilutions such estimation might not be correct [44]. The original substance of the antibodies was produced in accordance with the current EU requirements of Good Manufacturing Practice for starting materials [45] by AB Biotechnology (Edinburgh, UK).

(2) Lactose saturated with water-ethanol solution free from highly diluted Abs.

(3) Lactose not subjected to any treatment.

All the lactose powders tested were provided by OOO "NPF "Materia Medica Holding" (Russia) in an encoded form (the samples were decoded after analysis of all the results obtained). The sampling of powders for the study was carried out in accordance with the internal sampling procedure that meets the pharmacopoeial requirements for sampling of crude drugs and medicinal products, ensuring uniformity of samples and excluding their contamination.

For experiments, $10 \% \mathrm{w} / \mathrm{w}$ aqueous solutions of the three abovementioned types of lactose powders were used. The solutions were prepared using deionized water obtained using a Milli-Q water purification system (Merck Millipore, Germany) with a conductivity of $18.3 \mathrm{M} \Omega \cdot \mathrm{cm}$. Dissolution was carried out at room temperature.

The solution of lactose saturated with a solution of highly diluted antibodies to IFN- $\gamma$ is hereinafter referred to as "HD Abs to IFN- $\gamma$ "; the solution of lactose saturated with water-ethanol solution free from highly diluted Abs is hereinafter referred to as "Saturation control"; the solution of lactose not subjected to any treatment is hereinafter referred to as "Intact lactose".

\subsection{Terahertz Time-Domain Spectroscopy (THz-TDS)}

THz-TDS is the main experimental approach used in the present study. Spectra were measured on a TPS Spectra 3000 spectrometer (TeraView Limited, Cambridge, UK) in a wavenumber range from 1.2 to $120 \mathrm{~cm}^{-1}$ with a resolution of $4 \mathrm{~cm}^{-1}$. Dynamic rangewas $40-80 \mathrm{~dB}$ in the specified spectral range. Each spectrum was obtained by averaging over 3600 scans. In the course of measurements, the spectrometer was purged with dried air obtained using a Parker Balston 74-5041 FT-IR Purge Gas Generator (Parker Hannifin Corporation, Haverhill, MA, USA).

The spectra of all the solutions studied were measured in two identical cuvettes with different distances between the windows: $50.7 \mu \mathrm{m}$ and $103.7 \mu \mathrm{m}$. Teflon gaskets were installed between the windows. After being filled with the sample, the cuvettes were hermetically closed with two screws with PTFE gaskets. The windows of the cuvettes are made of single-crystal quartz which has a high transmittance in the specified frequency range. Both cuvettes with the specified thickness were assembled before the cycle of conducted measurement and were not disassembled until the full completion of the experimental part of this work. Thus, the specified thickness can be considered standardized with an accuracy of $\pm 0.1 \mu \mathrm{m}$.

The exact distances between the windows were determined in an interferometric manner after the cuvettes were assembled. For this purpose, the transmittance spectra were measured for empty cuvettes in the range of $4000-8000 \mathrm{~cm}^{-1}$ using an IR spectrometer Nicolet 6700 . The spectra contain periodic bands which result from the interference of two waves: a wave transmitted through the cuvette without reflections, and a wave twice reflected between the windows of the cuvette. The distance 
between two adjacent interference fringes $\Delta v$ on a wavenumber scale is related to the distance between the planes of the windows $(l)$ through an equation:

$$
l(\mu \mathrm{m})=\frac{5000}{\Delta v\left(\mathrm{~cm}^{-1}\right)}
$$

Prior to measuring, the temperature of the analyzed solutions was stabilized at $30 \pm 0.5^{\circ} \mathrm{C}$ by placing the cuvette into a temperature-controlled rack of 4000 Series High Stability Temperature Controller (Specac Ltd., Orpington, UK). After reaching the target temperature of the cuvette, a two-minute intermission was made prior to starting the measurement in order to stabilize the sample temperature and dry the optical part of the spectrometer.

Following sequential measurement of the sample spectra in two indicated cuvettes, the single-beam spectrum obtained using the cuvette with a distance between windows of $103.7 \mu \mathrm{m}$ was divided by a single-beam spectrum obtained in a cell with a distance between windows of $50.7 \mu \mathrm{m}$. Thus, a $53 \mu \mathrm{m}$ thick transmittance spectrum of the solution was obtained avoiding virtually all optical artifacts. In addition, taking into account the known difference in the thickness of the sample ( $53 \mu \mathrm{m})$ in two cuvettes, the refractive index spectrum from the differential spectrum of the wave phase was calculated.

In order to account for the experimental error, 6 test solutions $(10 \% \mathrm{w} / \mathrm{w})$ were prepared from each of the powders studied. For each of the solutions, the spectra were recorded four times.

Such number of observations is sufficient for evaluating the experimental error and comparing various solutions with special statistical methods described in the section "Statistical data analysis".

\subsection{Terahertz Spectra Analysis}

The spectra of aqueous solutions in the terahertz frequency domain generally do not contain clearly defined spectral bands; therefore, to obtain interpretable information, the spectra recorded need to undergo mathematical processing. First, on the basis of the transmittance spectra $\operatorname{Tr}(v)$ and the refractive index $n(v)$, we calculated the imaginary part of the complex dielectric permittivity function of the substance according to the following equation:

$$
\varepsilon^{\prime \prime}(v)=-\frac{\mathrm{n}(v) \ln \operatorname{Tr}(v)}{2 \pi v \Delta l}
$$

where $v$-wavenumber in $\mathrm{cm}^{-1}, \Delta l$-difference between the interwindow distances of the two cuvettes used (in the present study $\Delta l=0.0053 \mathrm{~cm}$ ).

After calculating the function $\varepsilon^{\prime \prime}(v)$, we implemented its decomposition into band components which are described in the literature and well-interpreted at the molecular level. To date, at least three water absorption bands with known molecular dynamics interpretation have been reliably established in the spectra of aqueous solutions in the terahertz frequency domain: (1) the Debye relaxation band with an absorption maximum of about $0.6 \mathrm{~cm}^{-1}$ [46]; (2) the intermolecular bending vibrational band with a maximum of absorption in the range $50-100 \mathrm{~cm}^{-1}$, and (3) the intermolecular stretching vibrational band with an absorption maximum of about $160-210 \mathrm{~cm}^{-1}[47,48]$. In the complex function $\varepsilon(v)$, these three bands are defined as follows:

$$
\varepsilon_{w}^{*}=\frac{\Delta \varepsilon_{1}}{1-i \omega \tau_{1}}+\frac{A_{1}}{\omega_{1}^{2}-\omega^{2}-i \omega \gamma_{1}}+\frac{A_{2}}{\omega_{2}^{2}-\omega^{2}-i \omega \gamma_{2}}+\varepsilon_{\infty}+i \frac{\sigma_{0}}{\varepsilon_{0} \omega}
$$

where $\omega-$ cyclic frequency associated with wave number $v$ through a relation $\omega=2 \pi \mathrm{c} v$, $\mathrm{c}$ - the speed of light; $\Delta \varepsilon$-contribution of the Debye relaxation of water molecules to the total dielectric response; $\tau$-relaxation time; $\omega_{1}$-resonant frequency of the intermolecular bending vibration; $\mathrm{A}_{1}$-contribution of the intermolecular bending band to the spectrum; $\gamma_{1}$ —width of this band; $\omega_{2}$-resonant frequency of the intermolecular stretching band; $\mathrm{A}_{2}$ - contribution of the intermolecular stretching band to the 
spectrum; $\gamma_{2}$-width of this band; the last component considers the ionic conductivity of the solutions, where $\sigma_{0}$ - conductivity at zero frequency, and $\varepsilon_{0}$-vacuum permittivity.

Selecting the imaginary part of function (3), we obtain $\varepsilon^{\prime \prime}(v)$ of the following form:

$$
\varepsilon^{\prime \prime}(v)=\frac{\Delta \varepsilon \omega \tau}{1+\omega^{2} \tau^{2}}+\frac{\mathrm{A}_{1} \omega \gamma_{1}}{\left(\omega_{1}^{2}-\omega^{2}\right)^{2}+\omega^{2} \gamma_{1}^{2}}+\frac{\mathrm{A}_{2} \omega \gamma_{2}}{\left(\omega_{2}^{2}-\omega^{2}\right)^{2}+\omega^{2} \gamma_{2}^{2}}+\frac{\sigma_{0}}{\varepsilon_{0} \omega}
$$

The model dielectric function (3) was used to describe the lactose solution, while we took into account only water bands and completely excluded from the consideration the spectral contribution of lactose itself. This is justified since the coefficient of water absorption in the studied region is 1.5-3 orders of magnitude greater than the absorption of lactose. If we take into account that the lactose content in the solution is $10 \%$, then the contribution of lactose to the absorption does not exceed $0.3 \%$. However, this does not mean that the spectrum of lactose solution is absolutely identical to the spectrum of water, since lactose can affect the characteristics of water itself through its hydration shells $[49,50]$.

We also note that model (3) is not the only correct one. In other cases, different models are used to describe the dielectric function of water in the terahertz region [51], including up to 3 relaxation and 3 vibrational bands. In this regard, the question arises: how many bands should be taken into account in this case? If we take into account a large number of bands, this will allow us to more accurately describe the experimental dielectric function. However, it would require taking into account a larger number of model parameters which, in turn, leads to a greater uncertainty in the values of these parameters in the calculation. If we take a small number of bands, then the model parameters are determined with a smaller spread. In this case, we will lose the ability to analyze other parameters. Thus, the information content of such a model will decrease. It is also important to mention that the interpretation of some bands from the terahertz region is still a subject of discussion [52-54]. Thus, even after obtaining the exact values of the parameters of the additional bands, we encounter uncertainty in the interpretation. In this study, an attempt was made to analyze aqueous solutions with very small differences in the spectra in the terahertz region. Therefore, the stability of the results obtained and the small scatter are extremely important for us. As we found out, this was achievable by taking into account three spectral bands. At the same time, the model we selected (3) is quite informative and allows to extensively characterize molecular structure and dynamics of the water phase, since each of these bands is associated with certain types of water dynamics at the intermolecular level. The model chosen (3) enables describing the properties of the solutions using defined physical parameters, such as the time of rotational relaxation of water molecules, the resonant frequency of intermolecular vibrations, and the contribution of particular molecular dynamic processes to the total dielectric response. It should be noted that two of the three bands chosen for the model have the peaks outside the measuring range: Debye relaxation band (at the low-frequency end of the range) and intermolecular stretching vibrational band (at the high-frequency end). However, these bands are very intense, and they quite strongly influence the features of spectra in the measured range [18]; therefore, it would be incorrect to ignore their impact.

Model (4) initially contains 9 parameters: $\tau, \Delta \varepsilon, A_{1}, \omega_{1}, \gamma_{1}, A_{2}, \omega_{2}, \gamma_{2}, \sigma_{0}$, the optimal values of which are to be determined. However, it is plausible to reduce the number of variable parameters in order to increase the reliability of fitting. First, the conductivity $\sigma_{0}$ was determined by direct measurements. Second, the two intermolecular bands (stretching and bending) have a similar molecular dynamic meaning - intermolecular vibrations of hydrogen-bonded water molecules. Therefore, one can omit some parameters of one of these two bands and focus on the analysis of the equivalent parameters of the other band. In this study, we used an approximation of consistency of the resonant frequency of the intermolecular stretching band $\omega_{2}$, setting it equal to $200 \mathrm{~cm}^{-1}$, which is a characteristic value for this type of vibration. The maximum of this band is located far from the analyzed spectral region, and its position varies slightly between aqueous solutions at the same temperature. Therefore, we did 
not include $\omega_{2}$ among the varied parameters. Third, when the binding of water molecules in the solution changes, the mean relaxation time $\tau$ may alter, which is related to a shift in the band maximum. Since we analyze the spectral region distant from the maximum of the Debye relaxation band $\left(0.6 \mathrm{~cm}^{-1}\right)$ and record only its high-frequency edge, a decrease/increase in the mean relaxation time $\tau$ is registered in our spectral range similarly to an increase/decrease in the amplitude $\Delta \varepsilon$. In other words, there is no need to vary both these parameters; therefore, the dielectric relaxation time $\tau$ was fixed at a value corresponding to the dielectric relaxation time of water $\left(\tau=7.315 \mathrm{ps}\right.$ at $30{ }^{\circ} \mathrm{C}$ [51]). The values of the other six model parameters: $\Delta \varepsilon, \omega_{1}, \mathrm{~A}_{1}, \gamma_{1}, \mathrm{~A}_{2}, \gamma_{2}$-were selected computationally using the fitting procedure.

The algorithm consisted in an extensive search of values of the model parameters and a search for a minimum value of deviation (s) of the experimental dielectric function (2) from the model function (4):

$$
\mathrm{s}=\frac{1}{\mathrm{~N}} \sqrt{\sum_{\mathrm{i}=1}^{\mathrm{N}}\left(\frac{\mathcal{\varepsilon}^{\prime \prime} \text { ex }\left(v_{\mathrm{i}}\right)-\varepsilon^{\prime \prime \bmod }\left(v_{\mathrm{i}}\right)}{\varepsilon^{\prime \prime \bmod }\left(v_{\mathrm{i}}\right)}\right)^{2}}
$$

where $\varepsilon^{\prime \prime \text { ex }}\left(v_{\mathrm{i}}\right)$ and $\varepsilon^{\prime \prime \bmod }\left(v_{\mathrm{i}}\right)$-values of the experimental and model functions $\mathcal{\varepsilon}^{\prime \prime}(v)$ for wavenumber $v_{i} ; \mathrm{N}-$ number of experimental points in a spectrum (in this study $\mathrm{N}=260$ ). A set of values of model parameters was considered optimal when the minimum value of $\mathrm{s}$ was attained. The fitting procedure can be considered sufficiently accurate if the value of s does not exceed 0.001 which was met in the present work.

Although the spectra were recorded in the range of $1.2-120 \mathrm{~cm}^{-1}$, for fitting the function $\varepsilon^{\prime \prime}(v)$ was used in the wavenumber range from 6 to $110 \mathrm{~cm}^{-1}$. This is due to the presence of spectral distortions at the edges of the spectral range of the spectrometer, or more specifically, the occurrence of a systematic error. This is a fairly stable distortion, i.e., the spectra of different samples can be reliably compared with each other, whereas calculating the physical parameters with the use of these parts of the spectrum is incorrect. The fitting procedure was sequentially implemented for each $\varepsilon^{\prime \prime e x}(v)$ function obtained from the experimental data.

Separately, it is important to indicate that we use only the imaginary part (4) to select the parameters of the model dielectric function (3). This is due, as mentioned above, to our desire to achieve the greatest reproducibility and the smallest spread in the values of the calculated parameters. The fitting of two functions (the real and imaginary parts of function (3)) inevitably leads to an increase in scatter which reduces the accuracy of the approach used.

\subsection{FTIR Spectroscopy}

In this paper, we also used the method of Fourier transform infrared spectroscopy (FTIR). The spectra were recorded using a spectrometer Nicolet 6700 (Thermo Fisher Scientific, Madison, WI, USA) in the transmittance mode in the wavenumber range of $6000-8000 \mathrm{~cm}^{-1}$, i.e., in the near infrared (NIR) region. This region includes the absorption band of the first overtone of intramolecular stretching vibrations of water molecules.

The overtone but not the main band was chosen due to the following reason. IR spectroscopy has been used to identify the differences in the average binding of water molecules between the analyzed solutions. The criterion of binding is the position of the absorption maximum of the NIR band which is directly related to the frequency of intramolecular vibrations of water molecules that depend on intermolecular binding. The shift of this band toward lower frequencies evidences an increase in the average energy of intermolecular hydrogen bonding, but normally the shift is very small, less than $1 \mathrm{~cm}^{-1}$. The overtone band is more suitable for analyzing the shift, since its frequency is approximately doubled as compared to the main vibrational band, and it is shifted for a double distance along the wavenumber scale resulting in a two-fold increase in the sensitivity of the method.

Each NIR spectrum was measured and averaged over 24 scans. The spectral resolution was $8 \mathrm{~cm}^{-1}$, while zero-extension of the interferogram was introduced in such a way that the distance between the 
points in a spectrum was $1 \mathrm{~cm}^{-1}$. A DTGS TEC detector with a $\mathrm{KBr}$ beam splitter was used. Prior to the Fourier transform, the Happ-Genzel apodization function was applied to the interferogram.

During the measurements, the optical part of the IR spectrometer was purged with air with reduced content of water vapor and $\mathrm{CO}_{2}$ using a dry air generator (Laboratory Gas Generator 74-5041). When recording the spectra, the temperature of the cuvette containing the sample was maintained at $30 \pm 0.5^{\circ} \mathrm{C}$.

The spectra of the studied solutions were recorded in a cuvette having various thickness with $\mathrm{CaF}_{2}$ windows. Single-beam spectra were recorded successively at two different thicknesses of the sample: $100 \pm 1 \mu \mathrm{m}$ and $450 \pm 1 \mu \mathrm{m}$. The spectrum of the sample in the thicker cuvette was divided by the spectrum of the sample in the thinner cuvette, allowing to obtain the transmittance spectrum of the analyzed solution with a thickness of $350 \pm 2 \mu \mathrm{m}$. Subsequently, a negative decimal logarithm was used to calculate the optical density spectrum from the transmittance spectrum. The spectra were smoothed using standard OMNIC software with averaging over 25 spectral points.

Each of the powders studied was used to prepare 3 replicates of solution $(10 \% \mathrm{w} / \mathrm{w})$ for analysis in order to allow for experimental error. Infrared spectra were recorded 5 times for each of the solutions. Such number of observations is sufficient for evaluating the experimental error and comparing various solutions with special statistical methods described in the Section 2.6

\subsection{Conductivity Measurement}

The conductivity of solutions $\sigma_{0}$ included in equation (4) was measured on a Zetasizer Nano ZS analyzer (Malvern, UK) using a mode for zeta potential measurement.

\subsection{Statistical Data Analysis}

The statistical significance of the differences between the groups was determined by variance analysis followed by comparison by the Tukey test using the $\mathrm{R}$ programming language (version 3.4.2) and was done in blind manner (tested samples were encoded). The level of statistically significant differences was set at $p<0.05$. The outliers were determined according to the plot of residuals of the linear model. The data are presented as "mean \pm standard error of the mean" ("M $\left.\pm \mathrm{SEM}^{\prime}\right)$.

\section{Results}

Figure 1 shows the representative spectra of transmittance (Figure 1a) and refractive index (Figure 1b) of the solutions for the Intact lactose. Corresponding spectra of solutions of the other samples (HD Abs to IFN- $\gamma$ and Saturation control) are very similar. Since the spectra are scarcely different from each other, in order to obtain the maximum information, in this study we implemented their decomposition into components according to the procedure described in Section 2.3. This procedure provided an opportunity to obtain statistically significant differences between the samples in a number of parameters calculated by the Equation (2), whose mean values are shown in Table 1.

Along with the analysis of terahertz spectra, the position of the maximum of the absorption band of the first overtone of intramolecular stretching vibrations of water molecules $2 v_{\max }$ in the NIR was determined (Figure 2). Table 2 contains $2 v_{\max }$ values for the solutions of the three tested samples of lactose.

As can be seen from Tables 1 and 2, based on the values of the parameters $\Delta \varepsilon, \omega_{1}, \gamma_{1}, \gamma_{2}$ and $2 \gamma_{\max }$, the solution of HD Abs to IFN- $\gamma$ exhibits statistically significant difference from the solutions of both the Saturation control and Intact lactose, while no statistically significant differences between them were revealed for the values of $A_{1}$ and $A_{2}$. The solutions of the Saturation control and Intact lactose showed a statistically significant difference only regarding the value of $\omega_{1}$. 

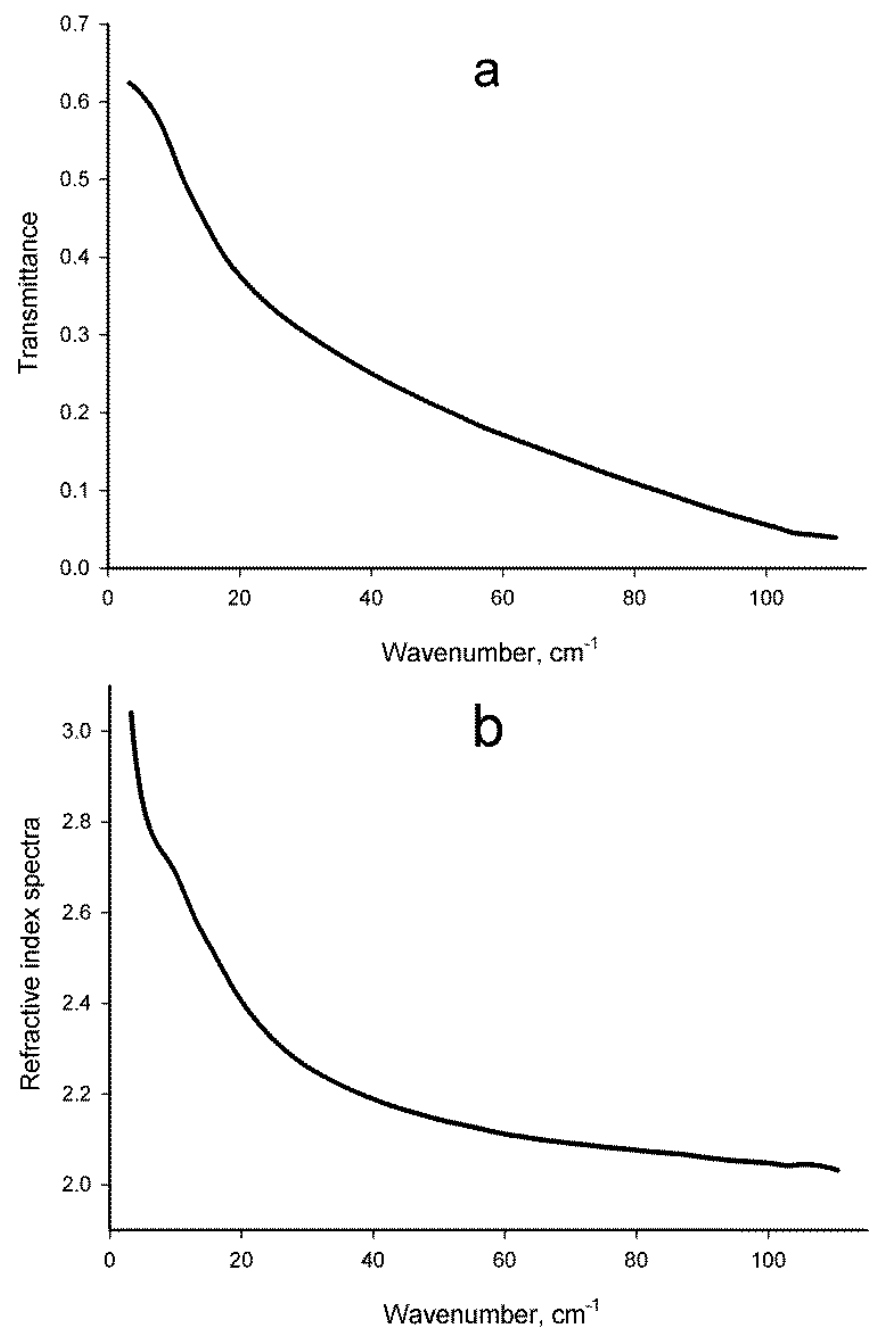

Figure 1. Representative transmittance (a) and refractive index (b) spectra of aqueous solutions of the Intact lactose sample.

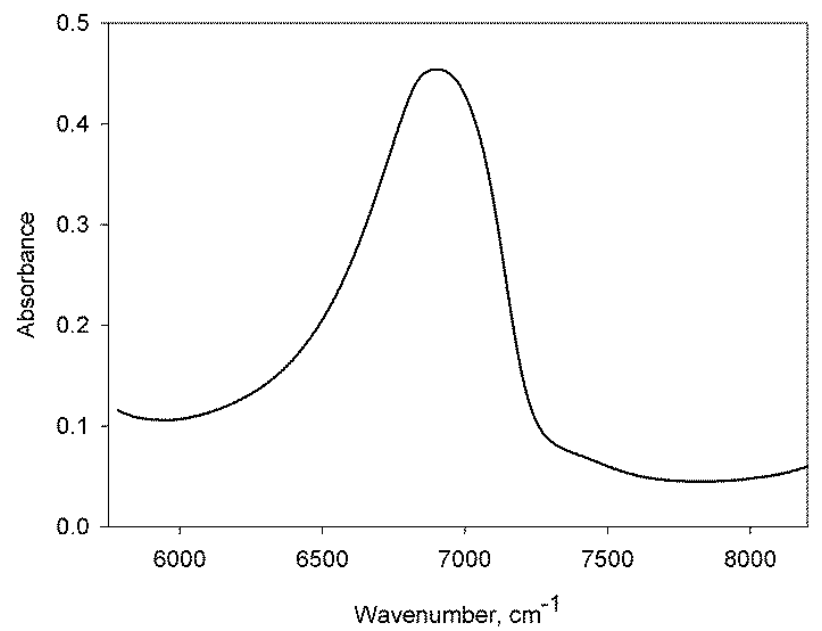

Figure 2. Representative band of $2 v_{\max }$ in the near infrared (NIR) spectrum of water solutions of the Intact lactose sample. 
Table 1. Results of terahertz time-domain spectroscopy data analysis: values of the calculated parameters of model (4) ${ }^{1}$.

\begin{tabular}{|c|c|c|c|}
\hline Parameter & Sample & Value & Statistically Significant Differences \\
\hline \multirow{3}{*}{$\Delta \varepsilon$} & HD Abs to IFN- $\gamma$ & $43.39 \pm 1.82$ & $*(p=0.031)$ \\
\hline & Saturation control & $48.33 \pm 0.95$ & $\&(p=0.025)$ \\
\hline & Intact lactose & $48.49 \pm 1.11$ & \\
\hline \multirow{3}{*}{$\omega_{1}\left(\mathrm{~cm}^{-1}\right)$} & HD Abs to IFN- $\gamma$ & $80.59 \pm 1.15$ & $*\left(p=1.7 \times 10^{-7}\right)$ \\
\hline & Saturation control & $88.93 \pm 0.94$ & $\&(p=0.012)$ \\
\hline & Intact lactose & $84.63 \pm 0.77$ & $\#(p=0.007)$ \\
\hline \multirow{3}{*}{$\gamma_{1}\left(\mathrm{~cm}^{-1}\right)$} & HD Abs to IFN- $\gamma$ & $510.1 \pm 0.4$ & $*\left(p=5.9 \times 10^{-4}\right)$ \\
\hline & Saturation control & $508.24 \pm 0.25$ & $\&(p=0.013)$ \\
\hline & Intact lactose & $508.73 \pm 0.33$ & \\
\hline \multirow{3}{*}{$\gamma_{2}\left(\mathrm{~cm}^{-1}\right)$} & HD Abs to IFN- $\gamma$ & $228.48 \pm 9.09$ & $*\left(p=7.2 \times 10^{-4}\right)$ \\
\hline & Saturation control & $189.71 \pm 7.04$ & $\&(p=0.007)$ \\
\hline & Intact lactose & $196.92 \pm 4.34$ & \\
\hline \multirow{3}{*}{$\mathrm{A}_{1} / 100\left(\mathrm{~cm}^{-2}\right)$} & HD Abs to IFN- $\gamma$ & $247.4 \pm 4.8$ & \multirow{3}{*}{-} \\
\hline & Saturation control & $245.1 \pm 3.5$ & \\
\hline & Intact lactose & $244.1 \pm 2.0$ & \\
\hline \multirow{3}{*}{$\mathrm{A}_{2} / 100\left(\mathrm{~cm}^{-2}\right)$} & HD Abs to IFN- $\gamma$ & $624.0 \pm 7.3$ & \multirow{3}{*}{-} \\
\hline & Saturation control & $625.2 \pm 14.8$ & \\
\hline & Intact lactose & $639.9 \pm 8.5$ & \\
\hline
\end{tabular}

1 The data are presented as "M $\pm \mathrm{SEM}$. The values of parameters $\mathrm{A}_{1}$ and $\mathrm{A}_{2}$ are reduced 100-fold for convenience of data analysis. * statistically significant difference of "HD Abs to IFN- $\gamma$ " group versus "Saturation control" group; \& statistically significant difference of "HD Abs to IFN- $\gamma$ " group versus "Intact lactose" group; \# statistically significant difference of "Saturation control" group versus "Intact lactose" group.

Table 2. Results of FTIR spectroscopy: the position of the maximum of the absorption band of the first overtone of intramolecular stretching vibrations of water molecules $2 v_{\max }$ in the NIR spectra of aqueous solutions of the analyzed lactose samples ${ }^{1}$.

\begin{tabular}{|c|c|c|}
\hline Sample & $2 v_{\max }\left(\mathrm{cm}^{-1}\right)$ & Statistically Significant Differences \\
\hline HD Abs to IFN- $\gamma$ & $6888.91 \pm 0.47$ & $*(p=0.035)$ \\
\hline Saturation control & $6890.39 \pm 0.41$ & $\&(p=0.020)$ \\
\hline Intact lactose & $6890.52 \pm 0.33$ & \\
\hline
\end{tabular}

1 The data are presented as "M \pm SEM". * Statistically significant difference of "HD Abs to IFN- $\gamma$ " group versus "Saturation control" group; \& statistically significant difference of "HD Abs to IFN- $\gamma$ " group versus "Intact lactose" group.

\section{Discussion}

In this study, the THz-TDS method was first used for studying solutions that contain lactose saturated with HD Abs to IFN- $\gamma$. The rationale for this work lies in the published results that have characterized the solutions of HD Abs as a self-organizing dispersed system in which nano-scale objects are generated [55]. It was demonstrated that solution of the HD Abs may contain aggregates of original Abs with gas nanobubbles which survived after multiple dilutions due to the flotation effect [44]. These results are in line with the previously shown presence of nanoparticles of the starting material even at a very high dilution level [56]. As THz-TDS method is highly sensitive to the intermolecular interactions of the water molecules, such a procedure could be the assay of choice for the testing of highly diluted samples. 
From the approach proposed for the analysis of terahertz spectra, a number of parameters were obtained (Table 1), and for some of them the HD Abs to IFN- $\gamma$ statistically significantly differs from the control solutions. This fact indicates the high sensitivity and informative value of the proposed THz-TDS-based approach for analyzing this class of solutions.

In order to give an interpretation of the differences shown in this study, consideration should be given to the meaning of each band included into model (3). The relaxation band in the spectra reflects the Debye relaxation of water molecules in solution. This band is described by two parameters: relaxation time $\tau$ and amplitude $\Delta \varepsilon$. The parameter $\tau$ determines specific time of finding a water molecule in the bound state between the moments of hydrogen bond disruption and molecule reorientation. The parameter $\Delta \varepsilon$ reflects the contribution of the relaxation process to the total dielectric response. In this study, only $\Delta \varepsilon$, and not the parameter $\tau$ (it was fixed), was calculated in the fitting procedure. An increase of $\tau$ (a shift of the band maximum to the low-frequency region of the spectrum) in the terahertz region was registered similarly to a decrease in the amplitude of $\Delta \varepsilon$. In the investigated spectral range, both trends (an increase of $\tau$ and a decrease of $\Delta \varepsilon$ ) are indistinguishable and indicate the same phenomenon: intensification of depolarization of water molecules in the solution, i.e., their enhanced binding. Therefore, in our model, the parameter $\Delta \varepsilon$ alone is sufficient for describing the depolarization processes.

The data in Table 1 show a statistically significant decrease in $\Delta \varepsilon$ for the HD Abs to IFN- $\gamma$ in comparison to both controls, suggesting an increased binding of water molecules in this solution. This conclusion is also supported by a statistically significant reduction in the frequency $2 v_{\max }$ obtained by IR spectroscopy in the HD Abs to IFN- $\gamma$ as compared to both controls (Table 2). As is known [57], a decrease in the frequency of intramolecular stretching vibrations indicates an increase in the intermolecular binding of water molecules in the solution.

A statistically significant decrease was observed in the resonant frequency of translational oscillations $\omega_{1}$ in the spectrum of the solution of lactose saturated with the HD Abs to IFN- $\gamma$ (Table 1) as compared to both controls. The indicated difference in $\omega_{1}$, when considered separately, can be interpreted as weakening of bonds between water molecules in the studied solution and, at first sight, this conclusion is in contradiction with the previous assumption. However, this apparent contradiction is resolved by taking into consideration the established differences in the parameters of the widths of the transmission bands $\gamma_{1}$ and $\gamma_{2}$, which will be described below.

A statistically significant increase in the values of $\gamma_{1}, \gamma_{2}$ in the HD Abs to IFN- $\gamma$, as compared to both controls, indicates a broadening of energy distributions of intermolecular (hydrogen) bonds in this solution. Importantly, the parallelism of this broadening tendency in the two transmission bands strengthens the reliability of this conclusion.

Regarding the apparent contradiction between the two conclusions mentioned above, in HD Abs to IFN- $\gamma$, intermolecular hydrogen bonds are strengthened, as evidenced by the decrease in $\Delta \varepsilon$; moreover. The weakening of intermolecular hydrogen bonds can also be recorded which is indicated by a decrease in $\omega_{1}$. This issue is resolved when taking into account the registered broadening of energy distributions of intermolecular hydrogen bonds in the HD Abs to IFN- $\gamma$. Apparently, different parameters of the bands of terahertz spectra exhibit various sensitivity to the strengthening and weakening of intermolecular bonds. When the energy distributions of intermolecular bonds are broadened, more molecules emerge both with stronger and weaker intermolecular bonds. In this case, the amplitude $\Delta \varepsilon$ as well as the frequency $2 v_{\max }$ show a stronger response to an increase in the energy of intermolecular bonds. Parameter $\omega_{1}$, on the contrary, is to a higher extent contributed to by molecules with weakened intermolecular energy. Thus, the previously used different spectral parameters allow analyzing the process of structural changes in solutions from different perspectives. Various levels of sensitivity of these parameters to the strengthening and weakening of hydrogen bonds may be related to the difference in the transition dipole moments in different types of vibrations (intramolecular and intermolecular). This issue deserves a separate thorough study within the framework of the theory of vibrational spectroscopy; however, this aspect is beyond the scope of the present investigation. 
Regarding the parameters $\Delta \varepsilon, \gamma_{1}, \gamma_{2}$ and $2 \gamma_{\max }$, for which statistically significant differences between the HD Abs to IFN- $\gamma$ and the control solutions were found, no statistically significant differences between the control solutions were observed, which in general increases the validity of the techniques used for sample testing. At the same time, the parameter $\omega_{1}$, which also differed between the control solutions, may be sensitive to minor changes in the solution state, i.e., a statistically significant difference in $\omega_{2}$ between the controls may indicate a certain physicochemical modification of the control lactose sample after saturation with water-alcohol solution which is likely mediated by technological reasons. Considering this, it can be concluded that the THz-TDS method is capable of tracking even minor differences in lactose samples and, after establishing proper standards, can be used to control the quality of manufactured drugs based on highly diluted substances.

It should be mentioned separately that the mathematical model for analysis of terahertz spectra proposed in this paper is not the only and ultimate one. It can be expanded and extended. For instance, other spectral bands from within the studied frequency range or beyond can be added for consideration, thereby expanding the set of parameters characterizing the analyzed system. Further developing this approach, one can add to the consideration the librational absorption band with a maximum of about $600 \mathrm{~cm}^{-1}$ [47], reflecting the rotational vibrations of water molecules bound by hydrogen bonds. Also, more recently, attention has been paid to another relaxation band of about $20 \mathrm{~cm}^{-1}$ [22,54,58], which can be considered as well. It is important to maintain a balance between the number of parameters taken into account and the stability of the fitting procedure, since too many parameters can, firstly, complicate calculations, and secondly, lead to ambiguities in the results obtained and difficulties in interpreting them.

Using the described approach, we demonstrate that the THz-TDS method has advantages over the IR spectroscopy method traditionally used to analyze structural properties of water solutions $[59,60]$. Terahertz spectroscopy, taking into account its location on the frequency scale, contains data on intermolecular dynamics in aqueous solutions. IR spectroscopy contains data on the intramolecular dynamics. At the same time, the intramolecular vibrations of the water molecule depend on the intermolecular binding of these molecules. This underlies an approach that allows us to evaluate the binding of water in various systems using IR spectra. That is, we can say that $\mathrm{THz}$ spectroscopy analyzes intermolecular characteristics directly, and IR spectroscopy does it indirectly. One of the main conclusions of our work is that $\mathrm{THz}$ spectroscopy provides significantly more information than IR spectroscopy, and the data obtained using both methods do not contradict each other. Let us consider the potential use of spectroscopy in an even shorter part of the electromagnetic scale (optical or UV range). The photons energies from these ranges correspond to transitions between the electron shells of molecules, which is about 3 orders of magnitude higher than the energies of intermolecular interactions. According to the Born-Oppenheimer approximation, the types of system dynamics that differ so much in energy can be considered independent with high accuracy. Therefore, approaches based on optical or UV spectroscopy are practically not applicable for analyzing the intermolecular properties of aqueous solutions and are not able to be informative in terms of the structural properties of aqueous solutions.

With THz-TDS information received by the IR spectroscopy can be significantly expanded. Thus, in addition to studying the intermolecular bonding of water molecules in solution (and the results of the THz-TDS method (parameter $\Delta \varepsilon$ ) are confirmed by IR spectroscopy $\left(2 v_{\max }\right)$ ), it is also possible to analyze the change in the energy distributions of intermolecular (hydrogen) bonds in solution (parameters $\gamma_{1}$ and $\gamma_{2}$ ) using our approach to the THz-TDS method. The results obtained in this study allow us to conclude that the THz-TDS method, when using the described approach, can be applied for analyzing the structural properties of solutions containing highly diluted antibodies.

An elucidation of the causes underlying the registered structural changes in the HD Abs to IFN- $\gamma$ requires a separate, more thorough study. It may be assumed that the changes in the structure and dynamics of water molecules in this solution can be mediated by a change in the parameters of hydrate shells of lactose molecules or by the formation of complexes consisting of water and lactose molecules, which are different from those in the control solutions. This may be due to at least two 
reasons. First, lactose has changed its physicochemical properties, for example, its conformation, as described in [61] in the process of saturation with highly diluted antibodies (specifically, since such a solution differs from a solution for saturation control). Second, it is reasonable to assume that nanoscale objects based on technologically processed (during dilution and vibration exposure) molecules of the original antibodies are present in the highly diluted antibody solution used for lactose saturation [44]. Apparently, lactose can accumulate these objects during saturation and release them during dissolution.

\section{Conclusions}

The paper describes the use of the terahertz time-domain spectroscopy for the physicochemical analysis of preparations of aqueous solutions of highly diluted antibodies. The proposed approach is based on the analysis of the dielectric function of water based on a three-band (Debye relaxation, intermolecular bending, and stretching vibrational bands) model. This model allows us to characterize these solutions by a number of parameters that have a clear molecular dynamic interpretation. Statistically significant differences between $\Delta \varepsilon, \gamma_{1}, \gamma_{2}$ parameters of an aqueous solution of lactose saturated with a solution of highly diluted antibodies to IFN- $\gamma$ and control solutions were established. This indicates a broadening of the energy distribution of intermolecular water bonds in a solution of preparations containing highly dilute antibodies. The findings based on the analysis of terahertz spectra were confirmed by the data obtained using such a widely recognized method as FTIR spectroscopy: both methods can register more strongly bound water molecules in the solution of lactose saturated with a solution of highly diluted antibodies to IFN- $\gamma$. However, THz-TDS provides significantly more information than IR spectroscopy. The proposed approach can be used for the indirect investigation of a substance present in an untraceable amount in aqueous solutions by measuring its effect on properties of water molecules.

Author Contributions: Conceptualization, N.P. and E.F.; methodology, N.P.; software, N.P.; validation, N.P., E.F.; formal analysis, N.P.; investigation, N.P.; resources, N.P. and E.F.; data curation, N.P.; writing-original draft preparation, N.P.; writing - review and editing, E.F.; visualization, N.P. and E.F.; supervision, N.P. and E.F.; project administration, N.P.; funding acquisition, N.P. All authors have read and agreed to the published version of the manuscript.

Funding: This project was funded through a formalized research contract between Institute of Cell Biophysics, Federal Research Center "Pushchino Scientific Center for Biological Research of the Russian Academy of Sciences", Russian Academy of Sciences and OOO "NPF "Materia Medica Holding".

Acknowledgments: This work was supported by the equipment of Optical Microscopy and Spectrophotometry core facility, ICB RAS, Federal Research Center "Pushchino Scientific Center for Biological Research of the Russian Academy of Sciences"

Conflicts of Interest: The authors declare that they personally and independently conducted research on the contract between Institute of Cell Biophysics, Federal Research Center "Pushchino Scientific Center for Biological Research of the Russian Academy of Sciences", Russian Academy of Sciences and OOO "NPF "Materia Medica Holding". Lactose powders were provided by OOO "NPF "Materia Medica Holding" under the author's request. Different versions of highly diluted antibodies to IFN- $\gamma$ are the substances (single or one among other components) for commercial drugs produced or produced and marketed by OOO "NPF "MATERIA MEDICA HOLDING". Patents on this substance belong to OOO "NPF "MATERIA MEDICA HOLDING". The authors declare no other conflict of interest. The funders took part in the design of the study; and had no role in the collection, analyses, or interpretation of data; in the writing of the manuscript, or in the decision to publish the results.

\section{References}

1. Penkov, N.V.; Yashin, V.; Belosludtsev, K.N. Hydration Shells of DPPC Liposomes from the Point of View of Terahertz Time-Domain Spectroscopy. Appl. Spectrosc. 2020. [CrossRef]

2. Markelz, A.G.; Roitberg, A.; Heilweil, E.J. Pulsed terahertz spectroscopy of DNA, bovine serum albumin and collagen between 0.1 and 2.0 THz. Chem. Phys. Lett. 2000, 320, 42-48. [CrossRef]

3. Yoneyama, H.; Yamashita, M.; Kasai, S.; Kawase, K.; Ueno, R.; Ito, H.; Ouchi, T. Terahertz spectroscopy of native-conformation and thermally denatured bovine serum albumin (BSA). Phys. Med. Biol. 2008, 53, 3543-3549. [CrossRef] 
4. Whitmire, S.E.; Wolpert, D.; Markelz, A.G.; Hillebrecht, J.R.; Galan, J.; Birge, R.R. Protein Flexibility and Conformational State: A Comparison of Collective Vibrational Modes of Wild-Type and D96N Bacteriorhodopsin. Biophys. J. 2003, 85, 1269-1277. [CrossRef]

5. Menikh, A.; MacColl, R.; Mannella, C.A.; Zhang, X.-C. Terahertz biosensing technology: Frontiers and progress. Chemphyschem 2002, 3, 655-658. [CrossRef]

6. Mickan, S.P.; Menikh, A.; Liu, H.; Mannella, C.A.; MacColl, R.; Abbott, D.; Munch, J.; Zhang, X.-C. Label-free bioaffinity detection using terahertz technology. Phys. Med. Biol. 2002, 47, 3789-3795. [CrossRef]

7. Menikh, A.; Mickan, S.P.; Liu, H.B.; MacColl, R.; Zhang, X.C. Label-free amplified bioaffinity detection using terahertz wave technology. Biosens. Bioelectron. 2016, 20, 658-662. [CrossRef]

8. Cherkasova, O.; Nazarov, M.; Shkurinov, A. Noninvasive blood glucose monitoring in the terahertz frequency range. Opt. Quantum Electron. 2016, 48, 217. [CrossRef]

9. Pickwell, E.; Wallace, V.P. Biomedical applications of terahertz technology. J. Phys. D Appl. Phys. 2006, 39, R301. [CrossRef]

10. Kawase, K.; Ogawa, Y.; Watanabe, Y.; Inoue, H. Non-destructive terahertz imaging of illicit drugs using spectral fingerprints. Opt. Express 2003, 11, 2549-2554. [CrossRef]

11. Strachan, C.; Rades, T.; Newnham, D.A.; Gordon, K.C.; Pepper, M.; Taday, P.F. Using terahertz pulsed spectroscopy to study crystallinity of pharmaceutical materials. Chem. Phys. Lett. 2004, 390, 20-24. [CrossRef]

12. Taday, P.F. Applications of terahertz spectroscopy to pharmaceutical sciences. Philos. Trans. A. Math. Phys. Eng. Sci. 2004, 362, 351-363. [CrossRef]

13. Zhao, H.; Tan, Y.; Zhang, R.; Shalaby, M.; Zhang, C.; Zhao, Y.; Zhang, X.-C. Ultrafast hydrogen bond dynamics of liquid water revealed by terahertz-induced transient birefringence. Light Sci. Appl. 2020, 9, 136. [CrossRef]

14. Mickan, S.P.; Shvartsman, R.; Munch, J.; Zhang, X.C.; Abbott, D. Low noise laser-based T-ray spectroscopy of liquids using double-modulated differential time-domain spectroscopy. J. Opt. B Quantum Semiclassical Opt. 2004, 6, S786-S795. [CrossRef]

15. Ebbinghaus, S.; Kim, S.J.; Heyden, M.; Yu, X.; Heugen, U.; Gruebele, M.; Leitner, D.M.; Havenith, M. An extended dynamical hydration shell around proteins. Proc. Nat. Acad. Sci. USA 2007, 104, 20749-20752. [CrossRef]

16. Heyden, M.; Tobias, D.J.; Matyushov, D.V. Terahertz absorption of dilute aqueous solutions. J. Chem. Phys. 2012, 137, 235103. [CrossRef]

17. Bye, J.W.; Meliga, S.; Ferachou, D.; Cinque, G.; Zeitler, J.A.; Falconer, R.J. Analysis of the hydration water around bovine serum albumin using terahertz coherent synchrotron radiation. J. Phys. Chem. A 2014, 118, 83-88. [CrossRef]

18. Penkov, N.V.; Shvirst, N.E.; Yashin, V.A.; Fesenko, E.E. On singularities of molecular relaxation in water solutions. Biophysics 2013, 58, 731-738. [CrossRef]

19. Penkov, N.V.; Shvirst, N.E.; Yashin, V.A.; Fesenko, E.E. Calculation of the portion of free water molecules in water solutions by means of spectral analysis. Biophysics 2013, 58, 739-742. [CrossRef]

20. Penkov, N.V.; Yashin, V.A.; Shvirst, N.E.; Fesenko, E.E., Jr.; Fesenko, E.E. On peculiarities of temperature dependences of water spectra in the terahertz frequency domain. Biophysics 2014, 59, 220-222. [CrossRef]

21. Penkov, N.V.; Yashin, V.A.; Fesenko, E.E., Jr.; Fesenko, E.E. Calculation of the amount of free water molecules in aqueous solutions by means of spectral parameters from the terahertz frequency domain taking into account processes of screening. Biophysics 2014, 59, 347-350. [CrossRef]

22. Penkov, N.; Shvirst, N.; Yashin, V.; Fesenko, E., Jr.; Fesenko, E. Terahertz spectroscopy applied for investigation of water structure. J. Phys. Chem. B 2015, 119, 12664-12670. [CrossRef]

23. Penkov, N.; Yashin, V.; Fesenko, E., Jr.; Manokhin, A.; Fesenko, E. A Study of the Effect of a Protein on the Structure of Water in Solution Using Terahertz Time-Domain Spectroscopy. Appl. Spectrosc. 2018, 72, 257-267. [CrossRef]

24. Kardash, E.V.; Ertuzun, I.A.; Khakimova, G.R.; Kolyadin, A.N.; Tarasov, S.A.; Wagner, S.; Andriambeloson, E.; Ivashkin, V.T.; Epstein, O.I. Dose-response effect of antibodies to S100 protein and cannabinoid receptor type 1 in released-active form in the light-dark test in mice. Dose Response 2018, 16. [CrossRef]

25. Don, E.S.; Emelyanova, A.G.; Yakovleva, N.N.; Petrova, N.V.; Nikiforova, M.V.; Gorbunov, E.A.; Tarasov, S.A.; Morozov, S.G.; Epstein, O.I. Dose-Dependent Antiviral Activity of Released-Active Form of Antibodies to Interferon-Gamma against influenza A/California/07/09(H1N1) in Murine Model. J. Med. Virol. 2017, 89, 759-766. [CrossRef] 
26. Don, E.; Van der Meide, N.; Egorov, V.; Putilovskiy, M.; Tarasov, S. The level of natural autoantibodies to IFN-gamma in varicella infection treated with antiviral drug Anaferon for children: A pilot study. Immunol. Lett. 2020, 222, 90-94. [CrossRef]

27. Ivashkin, V.T.; Poluektova, E.A.; Glazunov, A.B.; Putilovskiy, M.A.; Epstein, O.I. Pathogenetic approach to the treatment of functional disorders of the gastrointestinal tract and their intersection: Results of the Russian observation retrospective program COMFORT. BMC Gastroenterol. 2019, 20, 2. [CrossRef]

28. Khacheva, K.K.; Khakimova, G.R.; Glazunov, A.B.; Fateeva, V.V. Technologically Processed Highly Diluted Antibodies to $\mathrm{S} 100$ Protein in the Treatment of Neurotic Disorders: The Review. Anxiety Disorders; IntechOpen: London, UK, 2020.

29. Penkov, N.V. Peculiarities of the Perturbation of Water Structure by Ions with Various Hydration in Concentrated Solutions of $\mathrm{CaCl} 2, \mathrm{CsCl}, \mathrm{KBr}$, and KI. Phys. Wave Phen. 2019, 27, 128-134. [CrossRef]

30. Aguejouf, O.; Malfatti, E.; Belon, P.; Doutremepuich, C. Time related neutralization of two doses acetyl salicylic acid. Thromb. Res. 2000, 100, 317-323. [CrossRef]

31. Marotta, D.; Marini, A.; Banaudha, K.; Maharaj, S.V.; Jonas, W.B. Nonlinear effects of glutamate and $\mathrm{KCl}$ on glutamate toxicity in cultured rat cerebellar neurons. Int. J. Neurosci. 2003, 113, 491-502. [CrossRef]

32. Belougne-Malfatti, E.; Aguejouf, O.; Doutremepuich, F.; Belon, P.; Doutremepuich, C. Combination of two doses of acetyl salicylic acid: Experimental study of arterial thrombosis. Thromb Res. 1998, 90, $215-221$. [CrossRef]

33. Epstein, O. The spatial homeostasis hypothesis. Symmetry 2018, 10, 103. [CrossRef]

34. Penkov, N.V. Temporal Dynamics of the Scattering Properties of Deionized Water. Phys. Wave Phenom. 2020, 28, 135-139. [CrossRef]

35. Cherkasova, O.P.; Nazarov, M.M.; Konnikova, M.; Shkurinov, A.P. THz Spectroscopy of Bound Water in Glucose: Direct Measurements from Crystalline to Dissolved State. J. Infrared Millimeter Terahertz Waves 2020, 41, 1057-1068. [CrossRef]

36. Lee, Y.-S. Principles of Terahertz Science and Technology; Springer: New York, NY, USA, 2009.

37. Theuer, M.; Harsha, S.S.; Molter, D.; Torosyan, G.; Beigang, R. Terahertz Time-Domain Spectroscopy of Gases, Liquids and Solids. Chemphyschem 2011, 12, 2695-2705. [CrossRef] [PubMed]

38. Mickan, S.P.; Abbott, D.; Munch, J.; Zhang, X.-C. Noise Reduction in Terahertz Thin Film Measurement Using Double Modulated Differential Technique. Fluct. Noise Lett. 2002, 2, R13-R28. [CrossRef]

39. Mickan, S.P.; Lee, K.S.; Lu, T.M.; Munch, J.; Abbott, D.; Zhang, X.C. Double modulated differential THz-TDS for thin film dielectric characterization. Microelectron. J. 2002, 33, 1033-1042. [CrossRef]

40. Ferguson, B.; Zhang, X.-C. Materials for terahertz science and technology. Nat. Mater. 2002, 1, $26-33$. [CrossRef]

41. Son, J.-H. Terahertz Biomedical Science and Technology; CRC Press: Boca Raton, FL, USA, 2014.

42. Merbold, H.P. Terahertz Time-Domain Spectroscopy of Aqueous Systems in Reflection Geometry and Construction of Polarisation-Sensitive Photoconductive Terahertz Antennas. Master's Thesis, University of Freiburg, Freiburg, Germany, 2006.

43. Epstein, O.I. Media and Method for Treating Pathological Syndrome. U.S. Patent 7572441B2, 8 November 2009.

44. Bunkin, N.F.; Shkirin, A.V.; Ninham, B.W.; Chirikov, S.N.; Chaikov, L.L.; Penkov, N.V.; Kozlov, V.A.; Gudkov, S.V. Shaking-Induced Aggregation and Flotation in Immunoglobulin Dispersions: Differences between Water and Water-Ethanol Mixtures. ACS Omega 2020, 5, 14689-14701. [CrossRef]

45. EU. Directive 2004/27/EC of the European Parliament and of the Council of 31 March 2004 amending Directive 2001/83/EC on the Community code relating to medicinal products for human use. Off. J. Eur. Union 2004, $136,34-57$.

46. Von Hippel, A.R. The dielectric relaxation spectra of water, ice, and aqueous solutions, and their interpretation. II. Tentative interpretation of the relaxation spectrum of water in the time and frequency domain. IEEE Trans. Terahertz Sci. Technol. 1988, 23, 817-823. [CrossRef]

47. Walrafen, G.E.; Fisher, M.R.; Hokmabadi, M.S.; Yang, W.H. Temperature dependence of the low- and high-frequency Raman scattering from liquid water. J. Chem. Phys. 1986, 85, 6970. [CrossRef]

48. Hasted, J.B.; Husain, S.K.; Frescura, F.A.M.; Birch, J.R. Far-infrared absorption in liquid water. Chem. Phys. Lett. 1985, 118, 622-625. [CrossRef] 
49. Heugen, U.; Schwaab, G.; Bründermann, E.; Heyden, M.; Yu, X.; Leitner, D.M.; Havenith, M. Solute-induced retardation of water dynamics probed directly by terahertz spectroscopy. Proc. Natl. Acad. Sci. USA 2006, 103, 12301-12306. [CrossRef]

50. Heyden, M.; Bründermann, E.; Heugen, U.; Niehues, G.; Leitner, D.M.; Havenith, M. Long-Range Influence of Carbohydrates on the Solvation Dynamics of Waters Answers from Terahertz Absorption Measurements and Molecular Modeling Simulations. J. Am. Chem. Soc. 2008, 130, 5773-5779. [CrossRef]

51. Ellison, W.J. Permittivity of pure water, at standard atmospheric pressure, over the frequency range $0-25 \mathrm{THz}$ and the temperature range $0-100^{\circ} \mathrm{C}$. J. Phys. Chem. Ref. Data 2007, 36, 1-18. [CrossRef]

52. Agmon, N. Tetrahedral displacement: The molecular mechanism behind the Debye relaxation in water. J. Phys. Chem. 1996, 100, 1072-1080. [CrossRef]

53. Zasetsky, A.Y. Dielectric relaxation in liquid water: Two fractions or two dynamics? Phys. Rev. Lett. 2011, 107, 117601. [CrossRef]

54. Lyashchenko, A.K.; Novskova, T.A. Structural dynamics of water and its dielectric and absorption spectra in the range 0-800 $\mathrm{cm}^{-1}$. J. Mol. Liquids 2006, 125, 130-138. [CrossRef]

55. Ryzhkina, I.S.; Murtazina, L.I.; Kiseleva, Y.V.; Konovalov, A.I. Self-organization and physicochemical properties of aqueous solutions of the antibodies to interferon gamma at ultrahigh dilution. Dokl. Phys. Chem. 2015, 462, 110-114. [CrossRef]

56. Chikramane, P.S.; Kalita, D.; Suresh, A.K.; Kane, S.G.; Bellare, J.R. Why extreme dilutions reach non-zero asymptotes: A nanoparticulate hypothesis based on froth flotation. Langmuir 2012, 28, 15864-15875. [CrossRef] [PubMed]

57. Yukhnevich, G.V. Infrared Spectroscopy of Water; Nauka: Moscow, Russia, 1973. (In Russian)

58. Yada, H.; Nagai, M.; Tanaka, K. Origin of the fast relaxation component of water and heavy water revealed by terahertz time domain attenuated total reflection spectroscopy. Chem. Phys. Lett. 2008, 464, 166-170. [CrossRef]

59. Brubach, J.-B.; Mermet, A.; Filabozzi, A.; Gerschel, A.; Roy, P. Signatures of the hydrogen bonding in the infrared bands of water. J. Chem. Phys. 2005, 122, 184509. [CrossRef] [PubMed]

60. Laporta, M.; Pegoraro, M.; Zanderighi, L. Perfluorosulfonated membrane (Nafion): FT-IR study of the state of water with increasing humidity. Phys. Chem. Chem. Phys. 1999, 1, 4619-4628. [CrossRef]

61. Chan, B. Aqueous-Phase Conformations of Lactose, Maltose, and Sucrose and the Assessment of Low-Cost DFT Methods with the DSCONF Set of Conformers for the Three Disaccharides. J. Phys. Chem. A 2020, 124, 582-590. [CrossRef] [PubMed]

Publisher's Note: MDPI stays neutral with regard to jurisdictional claims in published maps and institutional affiliations.

(C) 2020 by the authors. Licensee MDPI, Basel, Switzerland. This article is an open access article distributed under the terms and conditions of the Creative Commons Attribution (CC BY) license (http://creativecommons.org/licenses/by/4.0/). 